\title{
Relação entre satisfação sexual e satisfação com a imagem corporal de mulheres obesas e mulheres submetidas à cirurgia bariátrica
}

\author{
Relations between sexual satisfaction and satisfaction with the body image of obese women and \\ women undergoing bariatric surgery \\ Relación entre la satisfacción sexual y la satisfacción con la imagen corporal de mujeres obesas y \\ mujeres operadas de cirugía bariátrica
}

Recebido: 18/11/2021 | Revisado: 26/11/2021 | Aceito: 04/12/2021 | Publicado: 14/12/2021

\author{
Grazielle Cassia Alves de Souza \\ ORCID: https://orcid.org/0000-0002-2564-7199 \\ Faculdade de Ciências Aplicadas, Brasil \\ E-mail: grazielle_cassia@yahoo.com.br \\ Aline Santarem Ernesto \\ ORCID: https://orcid.org/0000-0001-5175-5399 \\ Instituto de Psicologia e Sexologia Essência Rara, Brasil \\ E-mail: santaremernesto@gmail.com \\ Priscila Junqueira \\ ORCID: https://orcid.org/0000-0001-5175-5399 \\ Instituto de Psicologia e Sexologia Essência Rara, Brasil \\ E-mail: prij@uol.com.br \\ Francine Náthalie Ferraresi Rodrigues Queluz \\ ORCID: https://orcid.org/0000-0002-8869-6879 \\ Centro Universitário Adventista de São Paulo \\ E-mail: francine.queluz@gmail.com
}

\begin{abstract}
Resumo
O presente artigo teve como objetivo comparar a imagem corporal e satisfação sexual em mulheres obesas e mulheres submetidas a cirurgia bariátrica há pelo menos 8 meses; verificar qual a autopercepção de ambas as amostras em relação ao peso; verificar se há correlação entre a satisfação com a imagem corporal e a satisfação sexual entre os dois grupos. Participaram 258 mulheres em relacionamento afetivo/sexual, sendo 100 classificadas com obesidade grau II e 158 estavam em condição pós cirurgia bariátrica. Todas as participantes responderam os seguintes instrumentos: Questionário de Caracterização Sociodemográfica; Body Shape Questionnaire e a Escala de Satisfação Sexual para Mulheres (SSS-W). Para a análise da dados, foi realizada análise estatística descritiva, teste $\mathrm{t}$, ANOVA e teste de correlação de Pearson. Como resultado verificou-se que o escore de satisfação sexual no SSSW foi de 106,57(mulheres submetidas à cirurgia) e de 102,98 (obesas), não havendo diferença significativa ( $p>0,05)$. Na satisfação com a imagem corporal, dentre as mulheres com cirurgia, $47,46 \%$ foram classificadas como satisfeitas, $25,95 \%$ com leve insatisfação, $18,36 \%$ apresentaram moderada insatisfação e $8,23 \%$ grave insatisfação. Dentre as mulheres obesas $14 \%$ apresentaram satisfação, $15 \%$ insatisfação em nível leve, $43 \%$ moderada insatisfação e $28 \%$ grave insatisfação da imagem. Foi encontrada correlação negativa estatisticamente significativa $(r=0,40 \mathrm{p}=0,001)$ entre Satisfação Sexual e Imagem Corporal. Conclui-se que, independentemente do peso, há a tendência de quanto maior a preocupação da mulher com a imagem corporal menor será sua satisfação sexual.
\end{abstract}

Palavras-chave: Sexualidade; Imagem corporal; Obesidade; Cirurgia bariátrica; Mulheres.

\begin{abstract}
This article aimed to compare body image and sexual satisfaction in obese women and women undergoing bariatric surgery for at least 8 months; check the self-perception of both samples in relation to weight; to verify whether there is a correlation between satisfaction with body image and sexual satisfaction between the two groups. 258 women in an affective/sexual relationship participated, 100 of which were classified as having grade II obesity and 158 were in a condition after bariatric surgery. All participants answered the following instruments: Sociodemographic Characterization Questionnaire; Body Shape Questionnaire and the Sexual Satisfaction Scale for Women (SSS-W). For data analysis, descriptive statistical analysis, $t$ test, ANOVA and Pearson correlation test were performed. As a result, it was found that the sexual satisfaction score in the SSSW was 106.57 (women undergoing surgery) and 102.98 (obese), with no significant difference ( $p>0.05$ ). In terms of body image satisfaction, among women with surgery, $47.46 \%$ were classified as satisfied, $25.95 \%$ as mildly dissatisfied, $18.36 \%$ as moderately dissatisfied and $8.23 \%$ as severely dissatisfied. Among obese women, $14 \%$ were satisfied, $15 \%$ were mildly dissatisfied, $43 \%$ were moderately dissatisfied
\end{abstract}


and $28 \%$ were severely dissatisfied with their image. A statistically significant negative correlation $(r=0.40 \mathrm{p}=0.001)$ was found between Sexual Satisfaction and Body Image. It is concluded that, regardless of weight, there is a tendency that the greater the concern a woman has with her body image, the lower her sexual satisfaction will be.

Keywords: Sexuality; Body image; Obesity; Bariatric surgery; Women.

\section{Resumen}

Este artículo tuvo como objetivo comparar la imagen corporal y la satisfacción sexual en mujeres obesas y mujeres sometidas a cirugía bariátrica durante al menos 8 meses; comprobar la autopercepción de ambas muestras en relación al peso; para verificar si existe una correlación entre la satisfacción con la imagen corporal y la satisfacción sexual entre los dos grupos. Participaron 258 mujeres en una relación afectiva / sexual, 100 de las cuales fueron clasificadas como obesas de grado II y 158 se encontraban en una condición después de una cirugía bariátrica. Todos los participantes respondieron los siguientes instrumentos: Cuestionario de Caracterización Sociodemográfica; Cuestionario de Forma Corporal y Escala de Satisfacción Sexual para Mujeres (SSS-W). Para el análisis de los datos se realizó análisis estadístico descriptivo, prueba t, ANOVA y prueba de correlación de Pearson. Como resultado, se encontró que el puntaje de satisfacción sexual en el SSSW fue de 106.57 (mujeres operadas) y 102.98 (obesas), sin diferencia significativa ( $p>0.05$ ). En cuanto a la satisfacción con la imagen corporal, entre las mujeres operadas, el 47,46\% se clasificaron como satisfechas, el 25,95\% como levemente insatisfechas, el 18,36\% como moderadamente insatisfechas y el $8,23 \%$ como gravemente insatisfechas. Entre las mujeres obesas, el $14 \%$ estaba satisfecho, el $15 \%$ estaba levemente insatisfecho, el $43 \%$ estaba moderadamente insatisfecho y el $28 \%$ estaba severamente insatisfecho con su imagen. Se encontró una correlación negativa estadísticamente significativa $(r=0.40 \mathrm{p}=0.001)$ entre Satisfacción Sexual e Imagen Corporal. Se concluye que, independientemente del peso, existe la tendencia de que cuanto mayor sea la preocupación de una mujer por su imagen corporal, menor será su satisfacción sexual.

Palabras clave: Sexualidad; Imagen corporal; Obesidad; Cirugía bariátrica; Mujeres.

\section{Introdução}

A imagem corporal (IC) pode ser definida como a representação mental que se possui do esquema corporal, sendo multidimensional, uma vez que abrange tanto as percepções do tamanho do corpo quanto as experiências emocionais envolvidas nesta percepção (Ferreira \& Leite, 2002). Trata-se da experiência psicológica do indivíduo sobre a aparência e funcionamento do seu próprio corpo (Cash, 1993).

A IC é parte fundamental do mecanismo de identidade pessoal (Kakeshita \& Almeida,2006), sua estruturação ocorre pela integração do desenvolvimento do indivíduo como um todo, envolvendo a percepção, atitudes e subjetividade de cada sujeito (Coelho et al., 2015). Ademais, as influências socioculturais podem induzir ao desejo de um corpo tido como ideal (Lira et al., 2017), uma vez que a magreza para as mulheres, e um corpo atlético para os homens são expostos e exaltados pela mídia e, em convenções culturais, estar acima do peso considerado ideal pode fazer com que a pessoa não seja aceita completamente pelo grupo no qual está inserida (Segura et al., 2016). O culto ao corpo magro traz consigo a imagem de beleza, de poder e aceitação, não dando espaço para a diversidade de formas e corpos que existem. Especialmente para as mulheres, a preocupação com a IC está associada ao peso, o que não é observado nos homens (Xavier, 2014).

Sabe-se que pessoas obesas sofrem preconceito por essa condição (Segura et al., 2016), possuem menos oportunidade de serem aceitas no ambiente escolar (Gobbi et al., 2017) e até mesmo no contexto do mercado de trabalho (Araújo et al., 2018). Enquanto se convive com a imperatividade do corpo magro, há em contrapartida um aumento no consumo de alimentos hipercalóricos (Justo, 2016). No Brasil, houve um aumento de 60\% dos quadros de obesidade nos últimos 10 anos (Ministério da Saúde, 2017).

A obesidade é uma doença crônica multifatorial, caracterizada pelo acúmulo de gordura corporal que causa prejuízos à saúde do indivíduo. Os fatores relacionados podem ser genéticos, ambientais, sociais e emocionais, sendo que a obesidade pode também impactar em todos estes âmbitos da vida. A classificação da doença é feita, majoritariamente, por meio de medidas antropométricas, em especial o Índice de Massa Corpórea (IMC). O IMC pode ser calculado pela razão entre o peso do indivíduo e altura elevada ao quadrado $\left(\mathrm{kg} / \mathrm{m}^{2}\right)$. Por ser de fácil aferição, é atualmente a forma de medida mais utilizada, porém não é um dado absoluto para se avaliar a obesidade, pois não é capaz de identificar se a massa calculada é referente a gordura ou ao 
músculo (Associação Brasileira para o Estudo da Obesidade e da Síndrome Metabólica. Diretrizes Brasileiras de Obesidade [ABESO], 2016).

Segundo a Associação Brasileira para o Estudo da Obesidade e da Síndrome Metabólica (ABESO, 2016) classifica-se como sobrepeso o indivíduo com IMC entre 25 a $29 \mathrm{Kg} / \mathrm{m}^{2}$ e obeso com IMC maior ou igual a $30 \mathrm{~kg} / \mathrm{m}^{2}$. Dentro da classificação de obeso há ainda os graus de intensidade, sendo considerado grau de risco I o IMC entre 30 a $34,9 \mathrm{~kg} / \mathrm{m}^{2}$; obesidade grau II entre 35 a $39,9 \mathrm{~kg} / \mathrm{m}^{2}$ e obesidade grau III com IMC superior a $40 \mathrm{~kg} / \mathrm{m}^{2}$. O tratamento para a obesidade é composto de intervenção multidisciplinar realizada por médicos, nutricionistas e psicoterapeutas e cada indivíduo deve ser abordado individualmente a fim de conhecer seu histórico de doenças e comportamentos, uma vez que a mudança no estilo de vida e da relação com a comida são fundamentais para o sucesso do tratamento. Muitas vezes o tratamento multidisciplinar é associado ao farmacológico (ABESO,2016).

Quando não se obtém sucesso na perda de peso por meio das ferramentas citadas anteriormente, em indivíduos com obesidade grau III, ou grau II associada a comorbidades (diabetes, hipertensão, apneia, dentre outras correlacionadas ao sobrepeso) pode ser indicado tratamento cirúrgico, a cirurgia bariátrica. Por representar uma alternativa eficaz no tratamento da obesidade severa, esse procedimento tem sido cada vez mais utilizado no Brasil, tendo o número de cirurgias quadriplicado nos últimos 10 anos, segundo a Sociedade Brasileira de Cirurgia Bariátrica e Metabólica (SBCMB, 2016).

A obesidade severa implica em algumas limitações na vida de mulheres, por exemplo, na realização de atividades básicas da vida diária (andar, calçar sapatos, passar na roleta do ônibus, sentar-se em cadeiras), bem como na disposição para o trabalho e na presença de limitações na vida sexual (Castro et al., 2010). Destarte, a cirurgia bariátrica pode ser uma alternativa não somente para a melhoria do quadro de obesidade, mas para a melhora da vida social, laboral e sexual.

Apesar de o procedimento cirúrgico ser uma alternativa eficiente para a perda de peso, por ela acontecer de maneira rápida, pode haver uma mudança brusca na composição corporal do paciente, mas não em sua auto IC. A acomodação psicológica com a nova imagem não se realiza de forma tão abrupta, em consequência, é possível que o indivíduo, apesar da perda de peso, ainda se perceba como obeso (Lacerda et al., 2018). Além disto, é necessário que o indivíduo submetido à cirurgia bariátrica se adapte às mudanças implicadas pela perda de peso, que influenciam a saúde física, social, pessoal e, em especial, à vivência da sexualidade. Nas mulheres, a melhora da autoestima e confiança na IC corrobora um aumento da satisfação sexual, já que mulheres submetidas ao procedimento cirúrgico relataram melhora no desempenho sexual, bem como na interação com o parceiro (Castro et al., 2010; Souza et al., 2020).

A sexualidade é um aspecto central da natureza humana, que envolve questões de gênero, orientação, prazer, intimidade e reprodução. Ela pode ser experimentada e expressada de diversas maneiras, pelo ato ou por pensamentos, desejos, crenças, valores e relações sociais. Pode-se dizer que a sexualidade humana envolve a interação dos aspectos biológicos, sociais, psicológicos, culturais, históricos e religiosos (OMS, 2015). A satisfação sexual é um construto amplo, que pode ser entendido como o grau no qual a atividade sexual de uma pessoa corresponde aos seus ideais (Pechorro et al., 2009). Desse modo, é possível considerar que a satisfação sexual está relacionada não somente ao ato sexual, mas com a satisfação conjugal, realização de fantasias, carícias, atração física, bem-estar físico e satisfação consigo (Catão et al., 2010; Alberto, 2016).

A obesidade pode interferir na vivência e função sexual dos indivíduos, pois além de uma alteração da libido e disfunções hormonais, existe a preocupação com a aceitação do outro (Oliveira et al., 2017), com a aprovação e interesse pelo corpo fora do padrão, que por sua vez pode criar limitações nos relacionamentos afetivos e sexuais (Marcelino \& Patrício, 2011). Em estudos anteriores foi possível verificar uma relação entre a perda de peso e melhora na função sexual (Barros et al., 2015; Pichlerova \& Zmolikova , 2019), todavia não há um consenso sobre o tema (Kaneshiro et al., 2008; Silva et al., 2013). Em recente revisão, foi verificada que pode haver fortes indícios que corroboram para a perspectiva de uma relação entre as funções sexuais e satisfação sexual com a obesidade, todavia se carece de estudos que analisem a sexualidade e as implicações 
psicológicas da obesidade, tais como autoestima e imagem corporal (Esfahani \& Pal, 2018).

Desse modo, a presente pesquisa tem por objetivo: comparar a imagem corporal e satisfação sexual em mulheres obesas e mulheres submetidas a cirurgia bariátrica há pelo menos 8 meses; verificar qual a autopercepção de ambas as amostras em relação ao peso; verificar se há correlação entre a satisfação com a imagem corporal e a satisfação sexual entre os dois grupos.

\section{Metodologia}

\subsection{Participantes}

Participaram deste estudo 252 mulheres que estavam em algum tipo de relacionamento afetivo/sexual com um parceiro(a), há pelo menos 6 meses, com idade média de 36 anos ( $\mathrm{DP}=7,88$ ). Elas foram divididas em dois grupos: Grupo I = 98 mulheres obesas grau II ou III, e idade média de 34 anos (DP = 7,88); e Grupo II = 154 mulheres que tiveram significativa perda de peso e realizaram a cirurgia bariátrica há, no mínimo, 8 meses, com idade média de 37 anos (DP = 7,78). O índice de massa corpórea das participantes foi calculado com base no relato do peso e altura. O IMC médio das participantes obesas foi de 41,58 ( $\mathrm{DP}=4,738)$ e das participantes que se submeteram a cirurgia bariátrica de 26,44 ( $\mathrm{DP}=3,29)$.

Foram excluídas da amostra as participantes que não estavam em um relacionamento há mais de 6 meses, bem como dentre as participantes do grupo de obesas - as que não se enquadravam nos graus II e III. No grupo pós bariátrica, foram excluídas da amostra as mulheres que realizaram o procedimento há menos de 8 meses. Deste modo, além das 252 participações válidas, houve uma perda amostral de 74 respostas que não atenderam aos critérios estabelecidos de participação.

\subsection{Instrumentos}

Questionário de Caracterização da amostra. Foi elaborado um questionário de caracterização da amostra pelas autoras deste estudo, composto de questões cujo objetivo foi levantar dados relevantes para a identificação das participantes, como por exemplo, idade, status do relacionamento conjugal, peso, altura, além da percepção acerca do peso, no qual deveriam indicar se se sentiam abaixo, dentro do peso ideal, pouco acima do peso ou muito acima do peso.

Body Shape Questionnaire (BSQ). Trata-se de uma escala do tipo Likert, que contém 34 questões, que podem ser respondidas entre 6 variáveis dentro de um continuum, variando entre "nunca" até "sempre". O BSQ tem o objetivo de mensurar a insatisfação com a IC e apresenta bons índices de confiabilidade, com alfa de Cronbach de 0,97. O resultado pode ser mensurado por meio da soma dos pontos obtidos nas respostas, de 1 a 6 , sendo interpretados como ausência de insatisfação (pontuação até 110), leve insatisfação (entre 111 e 138), moderada insatisfação (de 139 a 167 pontos) e grave insatisfação (acima de 167) (Freitas, 2016).

Escala de Satisfação Sexual para Mulheres (SSS-W). Trata-se de uma escala do tipo Likert composta por 30 itens, que se subdividem em 5 fatores a serem analisados: contentamento, comunicação, compatibilidade, preocupação relacional e preocupação pessoal. A versão brasileira do instrumento possui bons índices de confiabilidade, com alfa de Cronbach de 0,91 . Os itens são possuem cinco possibilidades de resposta em que "discordo totalmente" corresponde a 5 pontos até o "concordo totalmente" que corresponde a 1, sendo essas pontuações invertidas nos itens: 1, 4, 5, 9, 10,11,12. O escore total é obtido pela soma dos itens, quanto maior a pontuação, maior a satisfação sexual (Catão et al., 2010).

\subsection{Procedimento}

Após a aprovação do Comitê de Ética em Pesquisa da Universidade São Francisco (parecer 2.635.033), foi realizada a divulgação da pesquisa em redes sociais, em especial em grupos de pessoas que tenham feito, ou tinham interesse pelo tema da cirurgia bariátrica. No convite era descrito que a pesquisa tinha público-alvo somente mulheres. As interessadas, ao acessarem o formulário online, colocaram sua identificação (iniciais do nome, RG e e-mail) e tiveram acesso ao Termo de Consentimento 
Livre e Esclarecido. Após a ciência e aceite do termo, as participantes foram direcionadas para participarem do estudo e responderam ao Questionário de Caracterização da Amostra e BSQ e SSS-W.

\subsection{Análise dos dados}

Os dados foram analisados pela estatística descritiva considerando o grupo de participantes em seu total e divididos pelas variáveis de interesse do estudo. Foram analisados os efeitos das variáveis idade, região onde reside e tempo de cirurgia por meio do teste estatístico t de Student e por meio da Análise de Variância (ANOVA). A associação entre satisfação sexual e a insatisfação com a IC foi verificada por meio da correlação de Pearson, no qual as magnitudes da correlação serão consideradas da seguinte maneira: $r=0,1$ até 0,3 (fraco); $r=0,4$ até 0,6 (moderado); $r=0,7$ até 1 (forte) 48 . Foi realizada também a verificação do tamanho do efeito por meio do $\mathrm{d}$ de Cohen, no qual as magnitudes de menores que 0,19 são consideradas insignificantes, 0,20 a 0,49 pequena, 0,50 - 0,79 média, 0,80 - 1,29 grande e acima de 1,30 muito grande (Espírito Santo et al., 2017).

\section{Resultados}

Em relação satisfação com a IC em mulheres obesas e mulheres submetidas à cirurgia bariátrica, pôde-se verificar uma diferença significativa, pois no grupo 1, composto por mulheres obesas, $70,41 \%$ da amostra apresenta um grau de insatisfação com a IC de moderado a grave, enquanto no grupo 2, de mulheres que se submeteram à cirurgia bariátrica há pelo menos 8 meses, $25,97 \%$ da amostra se enquadra nessa mesma faixa de insatisfação. O resultado descritivo de cada amostra pode ser verificado na Tabela 1, bem como o resultado considerando a amostra geral.

Tabela 1. Resultado BSQ considerando os dois grupos e amostra total.

\begin{tabular}{lllllll}
\hline & Grupo 1 & \% Grupo 1 & Grupo 2 & \% Grupo 2 & Total & \% total \\
\hline Nenhuma insatisfação & 14 & 14,29 & 73 & 47,40 & 87 & 34,52 \\
Leve insatisfação & 15 & 15,31 & 41 & 26,62 & 56 & 22,22 \\
Moderada insatisfação & 42 & 42,86 & 28 & 18,18 & 70 & 27,78 \\
Grave insatisfação & 27 & 27,55 & 12 & 7,79 & 39 & 15,48 \\
\hline
\end{tabular}

Fonte: Autores.

Na Tabela 2 é possível verificar a comparação da média dos dois grupos quanto à satisfação com a IC. O escore médio no BSQ foi de 146,90, que aponta moderada insatisfação com a IC, foi encontrado entre as participantes do grupo 1, no grupo 2 o escore médio foi 111,81, que indica leve insatisfação com a imagem. Em relação à satisfação sexual, é possível verificar que o escore médio no SSS-W foi de 102,64 entre as mulheres do grupo 1 e de 106,23 entre as mulheres do grupo 2. Não houve diferença significativa entre os dois grupos $(p=0,324)$.

Sobre as subescalas do SSS-W, levando-se em consideração os dois grupos, o escore médio em todos os grupos foi acima de 15, em uma pontuação máxima de 30, o que indica certo grau de satisfação em todos os quesitos. Nas categ orias de comunicação, compatibilidade e preocupação relacional não houve diferença significativa entre as duas amostras ( $p>0,05)$, já em relação ao contentamento e preocupação pessoal houve diferença estatisticamente significativa entre os dois grupos da amostra $(\mathrm{p}<0,05)$. 
Tabela 2. Escore médio no BSQ e SSS-W considerando as duas amostras

\begin{tabular}{lllll}
\hline & Grupo 1 & Grupo $2 p$ & $d$ de Cohen \\
\hline BSQ total & 146,9 & 111,81 & $0,001 *$ & N/A \\
SSS-W total & 102,64 & 106,23 & 0,324 & N/A \\
SSS-W Comunicação & 22,653 & 21,78 & 0,318 & N/A \\
SSS - W: Compatibilidade & 22,327 & 22,1 & 0,792 & N/A \\
SSS-W: Contentamento & 17,95 & 19,3 & 0,025 & 0,292 \\
SSS-W P. relacional & 18,653 & 19,9 & 0,239 & N/A \\
SSS-W: P Pessoal & 21,031 & 23,13 & 0,034 & 0,275 \\
\hline \multicolumn{5}{c}{$* 0,05$. Fonte: Autores. } \\
\end{tabular}

Ao comparar a autopercepção com o IMC das participantes foi possível constatar que $27 \%$ das mulheres que possuíam IMC que indicaria peso normal, se consideraram ao menos um pouco acima do peso. Já 100\% das participantes com grau de obesidade III, se consideram muito acima do peso, conforme os resultados apresentados na tabela 3.

Tabela 3. IMC comparado a percepção do peso das participantes.

\begin{tabular}{|c|c|c|c|c|}
\hline IMC & Muito acima do peso* & Pouco acima do peso* & Peso Ideal* & Abaixo do peso ideal* \\
\hline Entre 18,6 e 24,9 & 3,390 & 23,729 & 66,102 & 6,780 \\
\hline Entre 25,0 e 29,9 & 5,263 & 67,105 & 27,632 & - \\
\hline Entre 30,0 e 34,9 & 31,818 & 63,636 & 4,545 & - \\
\hline Entre 35,0 e 39,9 & 82,353 & 17,647 & - & - \\
\hline Acima de 40,00 & 100 & & & \\
\hline
\end{tabular}

*Resultado em porcentagem (\%) considerando amostra total. Fonte: Autores.

Sobre a correlação entre a satisfação com a IC, satisfação sexual e peso obteve-se o resultado apresentado a seguir na Tabela 4 .

Tabela 4. Correlação de Pearson entre BSQ, SSS-W, e peso e percepção acerca do peso (se considerar acima do peso).

\begin{tabular}{lllll}
\hline & BSQ - Total & SSS-W - Total & Peso & $\begin{array}{l}\text { Se considera acima do } \\
\text { peso }\end{array}$ \\
\hline BSQ - Total & 1,000 & $-0,404$ & $-0,397$ & 0,570 \\
SSSW - Total & $-0,404$ & 1,000 & $-0,005$ & $-0,138$ \\
Peso & $-0,397$ & $-0,005$ & 1,000 & $-0,761$ \\
Se considera acima do peso & 0,570 & $-0,138$ & $-0,761$ & 1,000 \\
\hline
\end{tabular}

Fonte: Autores.

Baseado nos resultados obtidos foi possível verificar que houve uma correlação negativa significativa entre insatisfação com a IC e satisfação sexual, o que indica que quanto maior a insatisfação com a IC menor será a satisfação sexual. Ao relacionar o BSQ com o peso das participantes foi encontrada correlação positiva fraca. Já ao verificar a insatisfação com a IC e com se considerar acima do peso houve uma correlação positiva moderada, o que indica que quanto mais acima do peso a pessoa se 
considera, mais insatisfeita está com a IC. Também foi possível verificar uma fraca correlação negativa entre satisfação sexual e se considerar acima do peso, bem como uma correlação positiva forte entre peso e se considerar acima do peso, conforme tabela 4. Não houve correlação significativa entre o peso e a satisfação sexual.

\section{Discussão}

Neste estudo evidenciou-se que, independentemente do peso, há a tendência de quanto maior a preocupação da mulher com a imagem corporal menor será sua satisfação sexual. Ao analisar o construto de IC, conforme já descrito, é importante considerar que o meio sociocultural enaltece o corpo magro e atlético e marginaliza os demais corpos fora deste padrão, em especial quando as pessoas estão acima do peso e são, ao mesmo tempo, mulheres (Neves \& Mendonça, 2014). Isso pôde ser verificado no presente estudo ao se constatar que as mulheres obesas apresentaram maior insatisfação com a IC, se comparadas com as que já tiveram significativa perda de peso.

Há um resultado importante ao considerar que quase metade da amostra de mulheres que submeteram a cirurgia bariátrica não possui insatisfação com a IC, enquanto menos de 15\% da amostra de mulheres obesas se enquadrou como satisfeita. Pode-se pensar que a cirurgia bariátrica, para além dos benefícios à saúde metabólica, pode ser associada a consequências psíquicas e sociais positivas para parte das mulheres submetidas ao procedimento (Simões \& Brandão, 2020; Erden et al., 2015; Esfahani \& Pal, 2018). Todavia deve-se considerar que, apesar da significativa melhora na IC apresentada em estudos que analisam o construto em mulheres antes e depois da cirurgia há resultados que demonstram que boa parte de mulheres submetidas à bariátrica ainda se sentem insatisfeitas (Lacerda et al., 2018; Simões \& Brandão, 2020). Resultado semelhante ao encontrado no presente estudo pois percebe-se que, apesar de prevalência no grupo 1, em ambos os grupos houve uma insatisfação da imagem, indicando que as participantes, a despeito do peso, se sentem insatisfeitas com a IC.

Diante desses resultados, é possível verificar a complexidade da avaliação da IC, pois envolve facetas que têm sido estudadas em diversos outros campos científicos, tais como da educação física, nutrição e ciências sociais (Sousa Ribeiro et al., 2020; Oliveira et al., 2019; Le Breton, 2007). Devido aos resultados presentes neste estudo, e na literatura, é possível identificar a necessidade de uma abordagem com maior aprofundamento interdisciplinar para a melhor compreensão do tema, bem como auxiliar o indivíduo, seja ela obeso - no qual terá de lidar com as implicações sociais e psicológicas do estigma da obesidade bem como do paciente pós cirurgia bariátrica que terá de lidar com a mudança corporal e de hábitos.

Considerando os resultados encontrados quanto a satisfação sexual, foi possível perceber que, apesar da pontuação ter sido maior entre as mulheres que já tiveram uma significativa perda de peso, a diferença entre as duas amostras não foi significativa, visto que de uma escala de 0 a 150, houve uma diferença de menos de quatro pontos entre os grupos. Uma hipótese para este resultado é que, apesar de na literatura ser comumente possível encontrar resultados que associem obesidade a disfunções sexuais e que apontam a melhora do funcionamento sexual após a perda de peso (Barros et al., 2015; Pichlerova \& Zmolikova , 2019), há, em menor número pesquisas que, assim como o presente estudo, não constataram diferenças significativas no comportamento sexual e frequência de relações entre de mulheres obesas comparadas com uma amostra de mulheres com peso normal (Kaneshiro et al., 2008; Silva et al., 2013). Segundo essas pesquisas, isto acontece pela satisfação sexual estar relacionada a outros fatores tais como satisfação conjugal, comunicação com o parceiro e as atitudes e crenças sociais a respeito da liberdade sexual (Erden et al., 2015).

O presente estudo corrobora para a hipótese de que a satisfação sexual pode não estar relacionada ao peso do indivíduo, pois além da relação não significativa encontrada, a escala utilizada para aferir a satisfação sexual leva em consideração os fatores mencionados nos estudos de Erden et al (2015). Ao se analisar as subescalas do SSS-W, pode-se verificar que o contentamento refere-se a satisfação no âmbito sexual e emocional do relacionamento; comunicação diz respeito a discussão de questões sexuais e emocionais; compatibilidade: entre o desejo sexual percebido, crenças e valores sexuais e atitudes e 
similaridades percebidas do casal; preocupação relacional: percepção subjetiva a respeito de desconfortos e preocupações no relacionamento interpessoal sexual; preocupação pessoal: grau de desconforto sobre preocupações pessoais no âmbito sexual (Catão et al., 2010). Dessa maneira, considera-se que a sutil diferença nos dois grupos possa estar relacionada à qualidade da relação com o parceiro e demais fatores considerados para a avaliação da satisfação sexual.

A partir dessa perspectiva, verifica-se a necessidade de uma abordagem que considere também os aspectos sociais inerentes a nossa cultura, uma vez que a aceitação social promovida pelo movimento trouxe avanços não somente na discussão do tema, mas na qualidade de vida dessa população, inclusive na vivência da sexualidade das mulheres obesas (Neves \& Mendonça, 2014; Souza et al. 2020). É possível verificar também que uma porcentagem significativa das mulheres do grupo 1 que se consideraram muito acima do peso não possuía efetivamente um IMC que caracterizasse essa percepção. Isso pode ter relação com uma superestima da IC em pacientes em pós-operatório recente, conforme verificado no estudo de Moura (2018). Essa tendência foi notada em pacientes que realizaram a cirurgia há menos de seis meses no estudo citado, todavia na presente pesquisa essa tendência ocorreu em mulheres com no mínimo 8 meses de cirurgia. O mesmo resultado foi encontrado em pesquisas que comparavam grupos similares (Lacerda et al., 2018; Moura, 2018). Todavia não se pode generalizar essa conclusão uma vez que, na literatura é possível encontrar estudos no qual no decorrer do tempo após a cirurgia, parte da população se via menor do que realmente era (Rezende,2011). É possível pensar que ambas as formas de distorção de imagem podem ter relação com o período de adaptação do paciente pós bariátrico, pois com uma perda grande de peso em um curto período, pode ser difícil estimar o tamanho do corpo (Lacerda et al., 2018).

Ao analisar correlação negativa entre os dois construtos é cabível questionar o papel de demais variáveis, como a influência sociocultural e as pressões exercidas na população obesa como um fator que pode ser relevante para a vivência da sexualidade. Mariano et al (2014) relatam que apesar da obesidade ser uma das condições mais antigas da humanidade, atualmente há uma forte tendência de considerar a magreza como parâmetro para aceitação social, dessa maneira as pessoas que não se enquadram dentro do estereótipo considerado o ideal, tendem a se sentir fora do padrão estético e inibidos socialmente, o que dificultaria a relação com o parceiro e as experiências sexuais. De acordo com os resultados obtidos, pode-se considerar o fato de que tanto a obesidade quanto pressão com relação à IC podem ser relacionadas a outros fatores, como em estudos sobre a relação com à baixa autoestima (Barros et al., 2015), com o surgimento de sintomas depressivos e reações psicológicas adversas (Xavier \& Almeida, 2016). Diante deste contexto, é possível pensar na importância de um acompanhamento multiprofissional para os pacientes antes e depois da cirurgia, em especial o psicológico. Dessa maneira pesquisas posteriores seriam pertinentes para abordar com maior profundidade se haveria relação entre sintomas psicológicos, acompanhamento multiprofissional pós cirurgia e os construtos abordados na presente pesquisa.

\section{Conclusão}

Pode-se pensar que, no presente estudo, uma variável significante em relação à satisfação sexual seja a satisfação com a IC e não necessariamente o peso/obesidade, visto que não houve diferença significativa na satisfação sexual entre mulheres obesas e as submetidas a cirurgia bariátrica, ficando em maior evidência a disparidade entre a satisfação com a IC e a correlação negativa entre os construtos. Tanto a satisfação com a IC, quanto a satisfação sexual são construtos que levam em consideração diversos fatores sociais, biológicos e psicológicos para sua aferição, desse modo julga-se interessante em pesquisas futuras uma abordagem interdisciplinar sobre a temática. Cabe ressaltar que, apesar do crescente interesse no tema da sexualidade, ainda se carece de instrumentos validados para avaliação da satisfação sexual, bem como a escassez de estudos brasileiros que abordem a sexualidade feminina, principalmente de mulheres obesas ou que tiveram significativa perda de peso.

Apesar da homogeneidade da amostra e das limitações na abordagem de outras perspectivas socioculturais acerca do tema, pode-se considerar relevante o resultado da pesquisa, pois corrobora para mais evidências de que a obesidade pode não 
estar ligada diretamente à satisfação sexual, mas sim a preocupação com a IC, sendo necessário maior aprofundamento entre essa associação e as pressões sociais acerca da estética corporal, levando em consideração a influência sociocultural como também os movimentos emergentes de aceitação corporal. Cabe ressaltar que a satisfação sexual também é um construto complexo que pode ser influenciado não somente pela perspectiva abordada no estudo, como também por questões físicas e endócrinas. Ademais, o estudo se mostra relevante por contribuir com estudos futuros para se aprofundar em um tema relevante, vinculado não somente à obesidade, mas a qualidade de vida dessa população, além de contribuir para a verificação da necessidade de trabalhos futuros, com uma perspectiva interdisciplinar, tanto no entendimento quanto no tratamento da obesidade e da saúde sexual, levando-se em consideração não somente a relação com a imagem corporal, mas os aspectos fisiológicos envolvidos na função sexual e na perda de peso.

\section{Referências}

Alberto, K. (2016). Qualidade de vida, satisfação sexual e desempenho físico em adultos com fibrose cística. [Dissertação de Mestrado, Universidade Estadual de Campinas].

Almeida, A. S., \& Zanatta D. C., \& Rezende, F. F (2012). Imagem corporal, ansiedade e depressão em pacientes obesos submetidos à cirurgia bariátrica. Estudos em Psicologia (Natal), 17(1), 153-160. https://doi.org/10.1590/S1413-294X2012000100019

Araújo, L. S., Coutinho, M. P. L., Araújo-Morais, L. C., Simeão S. S. S., \& Maciel, S. C. (2018). Preconceito frente à obesidade: representações sociais veiculadas pela mídia impressa. Arquivos Brasileiros de Psicologia., 70(1), 69-85. http://pepsic.bvsalud.org/scielo.php?script=sci_arttext\&pid=S1809$52672018000100006 \& \operatorname{lng}=\mathrm{pt} \& \mathrm{nrm}=\mathrm{iso} \& \operatorname{lng}=\mathrm{pt}$

Associação Brasileira para o Estudo da Obesidade e da Síndrome Metabólica. (2016). Diretrizes Brasileiras de Obesidade. http://www.abeso.org.br/diretrizes Barros, L. M., Moreira, R. A. N., Frota, N. M., Araújo, T. M., \& Caetano, J. A. (2015). Qualidade de vida entre obesos mórbidos e pacientes submetidos à cirurgia bariátrica. Revista Eletrônica de Enfermagem, 17(2), 312-321. https://www.fen.ufg.br/revista/v17/n2/pdf/v17n2a15.pdf.

Cash, T. F. (1993). Body-image attitudes among obese enrollees in a commercial weight-loss program. Percept Motor Skills, 77(3), $1099-1103$.

Castro, M. R., Carvalho, RS. ., Ferreira, V. N., \& Ferreira, M. E. (2010) Função e imagem corporal: uma análise a partir do discurso de mulheres submetidas à cirurgia bariátrica. Revista Brasileira de Ciências e Esporte, 32(2-4). http://oldarchive.rbceonline.org.br/index.php/RBCE/article/view/689.

Catão, E., Rodrigues Jr., O. M., Viviani, D. H., Finotelli Jr., \& I, Silva F. R. C. S., (2010). Escala de satisfação sexual para mulheres: tradução, adaptação em estudo preliminar com amostra clínica. Boletim em psicologia, 60(133), 181-190. http://pepsic.bvsalud.org/scielo.php?script=sci_arttext\&pid=S000659432010000200005

Coelho, C. G., Giatti, L., Molina, M. D. C. B., Nunes, M. A. A., \& Barreto, S. M. (2015) Body image and nutritional status are associated with physical activity in men and women: The ELSA-Brasil study. International Journal Environment in Public Health. 12, 6179-6196.

Erden, S. C., Seyit, H., Yazisiz, V., Uyar, E. T., Akçakaya, R. O., Alis, H., Mehmet, D. G., \& Mehmet, M. (2015). Changes in Sexual Functions of Female Patients After Bariatric Surgery: Relationship with Body Image, Depression, and Anxiety. Bariatric Surgical Practice and Patient Care, 10(4), 150-5.

Esfahani, S. B., \& Pal S. (2018). Obesity, mental health, and sexual dysfunction: A critical review. Health Psychology Open. https://doi.org/10.1177/2055102918786867

Espírito Santo, H., \& Daniel, F. (2017) Calcular E Apresentar Tamanhos Do Efeito EM Trabalhos Científicos (1): As Limitações Do P<0, 05 Na Análise De Diferenças De Médias De Dois Grupos (Calculating and Reporting Effect Sizes on Scientific Papers (1): P<0.05 Limitations in the Analysis of Mean Differences of Two Groups). Revista Portuguesa de Investigação Comportamental e Social, 1(1), 3-16.

Ferreira M. C., \& Leite N. G. M. (2002) Adaptação e validação de um instrumento de avaliação da satisfação com a imagem corporal. Avaliação Psicoógica., 1(2), 141-149. http://pepsic.bvsalud.org/scielo.php?script=sci_arttext\&pid=S1677-04712002000200007\&lng=pt\&tlng=pt.

Freitas, B. I. (2016). Tratamento intensivo baseado na terapia de aceitação e compromisso para dificuldades relacionadas ao peso. [Dissertação de Mestrado, Pontifícia Universidade Católica do Rio Grande do Sul]. http://tede2.pucrs.br/tede2/handle/tede/6820

Gobbi, E., Greguol, M., Seron, B.B., \& Carraro, A. (2017). Um estudo exploratório da tendência "antiobesidade" entre professores e estudantes de educação física italianos. Movimento, 23(3), 963-974. https://seer.ufrgs.br/index.php/Movimento/article/view/73193.

Justo, A. M. (2016). Corpo e representações sociais: sobrepeso, obesidade e práticas de controle de peso [Tese de Doutorado, Universidade Federal de Santa Catarina]. https://repositorio.ufsc.br/handle/123456789/167973

Kakeshita, I. S., \& Almeida, S. S. (2006). Relação entre índice de massa corporal e a percepção da autoimagem em universitários. Revista de Saúde Pública, 40(3), 497-504. http://www.scielo.br/scielo.php?pid=S0034-89102006000300019\&script=sci_abstract\&tlng=pt

Kaneshiro, B., Jensen, J. T., Carlson, N. E., Harvey, S. M., Nichols, M. D., \& Edelman, A. B. (2008). Body mass index and sexual behavior., Obstetrics and Gynecology, 12(112), 586-592. 10.1097/AOG.0b013e31818425ec.

Lacerda, R. M. R., Castanha, C. R., Castanha, A. R., Campos, J. M., Ferraz, A. A. B., \& Vilar, L. (2018). Percepção da imagem corporal em pacientes submetidos à cirurgia bariátrica. Revista Brasileira de Cirurgia,45(2), e1793. http://www.scielo.br/scielo.php?script=sci_arttext\&pid=S0100$69912018000200160 \& \operatorname{lng}=\mathrm{pt} \& \mathrm{nrm}=\mathrm{iso} \& \operatorname{lng}=\mathrm{pt}$ 
Le Breton, D. (2007). A sociologia do corpo. Vozes.

Lira, A. G., Ganen, A. P., Lodi, A. S., \& Alvarenga, M. S. (2017). Uso de redes sociais, influência da mídia e insatisfação com a imagem corporal de adolescentes brasileiras. Jornal Brasileiro de Psiquiatria, 66(3), 164-171. http://www.scielo.br/scielo.php?script=sci_abstract\&pid=S004720852017000300164\&lng=en\&nrm=iso\&tlng=pt

Marcelino, L. F., \& Patrício, Z. M. (2011). A complexidade da obesidade e o processo de viver após a cirurgia bariátrica: uma questão de saúde coletiva. Ciência \& Saúde Coletiva, 16(12), 4767-4776. http://www.scielo.br/scielo.php?script=sci_arttext\&pid=S1413-81232011001300025

Mariano, M. L. L., Paula, M. A. B., Bassi, D. G., \& Paula, P. R. (2014). Cirurgia bariátrica: repercussões na sexualidade da pessoa obesa. Revista Brasileira de Cirurgia, 41(6), 412-420. http://www.scielo.br/scielo.php?pid=S0100-69912014000600412\&script=sci_abstract\&tlng=pt.

Martins, M. P., Abreu-Rodrigues, M. A. \& Souza, J. R. (2015). O uso da internet pelo paciente após cirurgia bariátrica: contribuições e entraves para o seguimento do acompanhamento multiprofissional. Arquivos Brasileiros de Cirurgia Diagnóstica.,28(1), 46-51. http://www.scielo.br/pdf/abcd/v28s1/pt_0102-6720-abcd28-s1-00046.pdf

Ministério da Saúde (2017). Obesidade cresce 60\% em dez anos no Brasil. http://www.brasil.gov.br/noticias/saude/2017/04/obesidade-cresce-60-em-dez-anosno-brasil

Moura, D. S. (2018). Qualidade de vida e percepção da imagem corporal de pacientes submetidos à cirurgia bariátrica. [Dissertação de Mestrado, Centro Universitário Franciscano]. http://www.tede.universidadefranciscana.edu.br:8080/bitstream/UFN-BDTD/605/5/Dissertacao_DeiseSilvaDeMoura.pdf

Neves, A. S., \& Mendonça, A. L. O. (2014). Alterações na identidade social do obeso: do estigma ao fat pride. Demetra., 9(3), 619-631. http://www.epublicacoes.uerj.br/index.php/demetra/article/view/9461/10922

Oliveira, E., Cardoso, D., \& Denari, F. (2017) O corpo humano como alimento para a sexualidade. Doxa: Revista Brasileira de Psicologia educacional, 19(1), 67-79.

Oliveira, T. C., Bering, T., Oliveira, J. R. T., \& Segri, N. J. (2019). Comportamento alimentar e imagem corporal em universitárias do curso de nutrição. Sigmae, $8(2), 771-778$.

Organização Mundial da Saúde - OMS (2015). Sexual health, human rights and the law. http://apps.who.int/iris/bitstream/10665/175556/1/9789241564984_eng.pdf?ua=1

Pechorro, P., Diniz, A., \& Vieira, R. (2009). Satisfação sexual feminina: relação com funcionamento sexual e comportamentos sexuais. Análise Psicológica, 23(1), 99-108. http://www.scielo.mec.pt/scielo.php?script=sci_arttext\&pid=S0870-82312009000100008.

Pichlerova, D., Bob, P., Zmolikova J,. Jitka, H., Radek, P., Matthew, K. L., Jiri, R. Tomas, F., \& Petr, W. (2019). Sexual Dysfunctions in Obese Women Before and After Bariatric Surgery. Medical Science Monitor., 25, 3108-3114. https://doi.org/10.12659/MSM.913614

Rezende, F. F. (2011). Percepção da imagem corporal, resiliência e estratégias de coping em pacientes submetidos à cirurgia bariátrica [Dissertação de Mestrado, Universidade de São Paulo, Ribeirão Preto]. http://www.teses.usp.br/teses/disponiveis/59/59137/tde-11052011-080701/pt-br.php

Segura, D. C. A., Corral, J. P., Wozniak, A. S., \& Vandresen, E. P. (2016). Análise da imagem corporal e satisfação com o peso em indivíduos submetidos à cirurgia bariátrica. Revista de Pesquisa em Saúde, 17(3), 170-174. http://www.periodicoseletronicos.ufma.br/index.php/revistahuufma/article/view/6791/4333

Silva, B. M., Rêgo, L. M., Galvão, M. A., Florêncio, T. M. M. T., \& Cavalcante, J. C. (2013). Incidência de disfunção sexual em pacientes com obesidade e sobrepeso. Revista do Colégio Brasileiro de Cirurgiões, 40(3), 196-202. https://doi.org/10.1590/S0100-69912013000300006

Simões, G., \& Brandão, I. (2020). Impacto da Cirurgia Bariátrica na Imagem Corporal e Saúde Mental. Revista Portuguesa De Psiquiatria E Saúde Mental, 5(4),51-61. https://doi.org/10.51338/rppsm.2019.v5.i4.90

Sociedade Brasileira de Cirurgia Bariátrica e Metabólica. (2016). Número de cirurgias bariátricas no Brasil cresce 7,5\% em 2016. Brasil. www.sbcbm.org.br/wordpress/numero-de-cirurgias-bariatricas-no-brasil-cresce-75-em-2016/

Sousa Ribeiro, S. A., Junior, G. D. B. V., Passos, R. P., Fileni, C. H., Lima, B. N., Oliveira, J. R. L., ... \& Militao, A. G. (2020); Imagem Corporal, Estado Nutricional E Nível De Atividade Física De Estudantes De Educação Física. Revista CPAQV-Centro de Pesquisas Avançadas em Qualidade de Vida-CPAQV Journal, 12(1). https://doi.org/10.36692/cpaqv-v12n1-9

Souza et al., Relações entre satisfação sexual e qualidade do relacionamento conjugal em universitárias. Psicologia para América Latina, (34), 133-141. http://pepsic.bvsalud.org/scielo.php?script=sci_arttext\&pid=S1870-350X2020000200005\&lng=pt\&tlng=pt.

Xavier, G. S., \& Almeida, S. S. (2016). The influence of bmi and psychological variables in the body size estimation among adult women. Psico,47(3): 179188. http://pepsic.bvsalud.org/pdf/psico/v47n3/02.pdf

Xavier, G. S. (2014). Avaliação da estimação da imagem corporal em mulheres adultas a partir de dois métodos perceptivos [Dissertação de Mestrado, Universidade de São Paulo, Ribeirão Preto]. http://www.teses.usp.br/teses/disponiveis/59/59137/tde-20052014-090852/en.php. 